Pecvnia, 6 (2008), pp. 175-193

\title{
El coste de oportunidad como determinante del autoempleo en la Unión Europea (UE 25)
}

Recibido: Abril 2008 Aceptado: Junio 2008
Luis Miguel Zapico Aldeano

luis-miguel.zapico.aldeano@unileon.es

Mariano Nieto Antolín

mariano.nieto@unileon.es

$M^{a}$ Felisa Muñoz Doyague

s.munoz@unileon.es

Universidad de León

Fac. de Ciencias Económicas y Empresariales Dpto. de Dirección y Economía de la Empresa Campus de Vegazana, $\mathrm{s} / \mathrm{n}$

24071 León (España)
Este trabajo pretende introducir en el análisis de la motivación de la actividad emprendedora el potencial efecto negativo del coste de oportunidad asociado a la misma. Para lograr este objetivo se aplicó un análisis de ecuaciones estructurales con la aproximación Partial Least Squares (PLS) sobre una muestra de ciudadanos de la Unión Europea agrupados por países, para el periodo 2004. Los resultados muestran que la motivación emprendedora presenta una estructura interna dual formada por dos componentes independientes, uno relacionado con el atractivo intrínseco del autoempleo
This paper tries to include the potential negative effect of the opportunity cost over the motivation analysis related to the entrepreneurial activity. According to this aim, a Structural Equation Modelling analysis with Partial Least Squares (PLS) approach was performed over a sample of European citizens clustered by country, within the period 2004. The results show that entrepreneurial motivation has a dual internal structure based on two independent components, one connected to the intrinsic attractiveness of self-employment and another one connected to the intrinsic attractiveness of the employee status. 
y otro relacionado con el atractivo intrínseco del estatus de empleado. Concretamente, los resultados indican una influencia directamente proporcional, sobre la intención emprendedora, de los elementos asociados al atractivo de ser empresario. También se verifica la relación inversamente proporcional de los elementos asociados al atractivo de ser empleado; siendo este efecto desmotivador de menor cuantía que el citado en primer lugar. Desde un punto de vista práctico, se plantea que las políticas de estímulo de la actividad emprendedora deberían tener en cuenta que no únicamente los ciudadanos con actitudes negativas hacia el autoempleo rechazan crear empresas, el atractivo del empleo por cuenta ajena es un determinante adicional significativo.

Palabras clave: Creación de empresas, motivación, autoempleo, empleado.

\begin{abstract}
Specifically, it is displayed a positive influence of items related to the attractiveness of selfemployment on entrepreneurial intentions. Also a negative relationship of items related to attractiveness of the employee status is verified; although, this negative motivation effect is smaller than the first positive one. From a practical point of view, it is proposed that entrepreneurship promotion policies should consider the fact that not only citizens with negative attitudes towards entrepreneurship reject to set up a business; the attractiveness of the employee status is an additional significant determinant.
\end{abstract}

Key words: Entrepreneurship, motivation, selfemployment, employee.

\section{INTRODUCCIÓN}

La creación de empresas es una actividad relacionada con el descubrimiento, evaluación y explotación de oportunidades para introducir nuevos productos y servicios, maneras de organizar mercados, procesos y materiales, a partir de esfuerzos organizativos inexistentes con anterioridad (Shane and Venkataraman 2000; Shane 2003). Consecuentemente, el aspecto central del fenómeno son las oportunidades de negocio, las cuales son un elemento objetivo, existen por sí mismas (Shane \& Venkataraman 2000: 220) y únicamente cuando un agente -emprendedorlas descubre y decide explotarlas se produce el acto de la creación empresarial y éstas tienen efectos económicos (Eckhardt \& Shane 2003: 336). Por otra parte, la prosperidad de los pueblos está unida al espíritu de empresa, a la vitalidad emprendedora (Cuervo 2003) y supone importantes beneficios, tanto económicos como sociales, pues no constituye únicamente una fuente de creación de empleo ${ }^{1}$, competitividad y crecimiento

Según los datos de la Comisión Europea (2004), un 31\% de los europeos -U. E. (25)-, actualmente no empleados por cuenta propia, probablemente recurran al autoempleo en los cinco próximos años. 
económico ${ }^{2}$, sino que también contribuye al alcance de objetivos sociales y al desarrollo del individuo ${ }^{3}$.

No obstante, y a pesar del fomento público de las actividades destinadas a la creación de empresas, aún es relativamente escaso el número de emprendedores ${ }^{4}$. Las razones para explicar el escaso interés de los individuos por la actividad emprendedora pueden buscarse en cualquiera de las fases del proceso: inexistencia de oportunidades relevantes, la falta de habilidades del individuo para llevar a cabo el proceso, la dificultad para acceder a los recursos necesarios, la incapacidad para combinar organizativa y estratégicamente esos recursos, etcétera. Así, es de esperar que la oportunidad en sí misma no sea el único motivador de la creación empresarial. La creación de empresas requiere la existencia de oportunidades, las cuales tienen un componente objetivo y un componente subjetivo; y es este componente subjetivo, fruto de las percepciones del individuo, el que influye en el descubrimiento, evaluación y explotación de la oportunidad (Shane 2003).

En este contexto nos planteamos analizar la motivación inicial de los individuos para crear una empresa: ¿por qué ante una misma situación dos individuos muestran conductas opuestas y uno quiere crear una empresa y otro no?. Dado que la motivación es una conducta dirigida y orientada hacia la consecución de metas, ¿cuál es la meta de un emprendedor?. Tradicionalmente los estudios sobre la motivación emprendedora han utilizado, fundamentalmente, rasgos de la personalidad del emprendedor y, en menor medida, características como los objetivos, expectativas y necesidades. No obstante, se ha relegado el estudio del efecto del "coste de oportunidad" de convertirse en empresario: el trabajo por cuenta ajena. Por ello, el propósito de este trabajo es realizar una comparación de los motivadores del trabajo por cuenta propia y ajena como determinantes de la intención de crear una empresa y, por motivos

2 Véase, entre otros, a Reynolds et al. (2005), Baumol (2004, 1993), Brown y Uljin (2004), Shane y Venkataraman (2000), Genescá et al. (2003), McGrath (1999), Hornaday (1992), Low y McMillan (1988).

Según los datos de la Comisión Europea (2004), los ciudadanos europeos -U. E. (25)- que preferirían estar en una situación de autoempleo justificarían esta decisión, principalmente, por la independencia o autosatisfacción que asocian a este estatus y al interés de las tareas que conlleva $(77 \%)$, seguido a mucha distancia por la consecución de mejores ingresos $(23 \%)$ y por otras razones diversas -oportunidad de negocio, coyuntura económica, etcétera-.

4 El panel Global Entrepreneurship Monitor, con datos relativos a 34 países, estimó que en el año 2004 solamente el 9,3\% de las personas en edad de trabajar son emprendedores (Instituto de Empresa, 2004). 
de eficiencia operativa, el análisis se centra en las percepciones de los ciudadanos de los 25 países europeos que formaban parte de la UE en 2004.

Los resultados del estudio muestran la influencia en la intención de crear una empresa tanto de los factores directamente relacionados con ser emprendedor como también por los factores directamente asociados con ser un empleado. Por otra parte, el efecto negativo de los factores relacionados con la actitud ante ser empleado es menor que el efecto positivo de los factores relacionados con la actitud ante ser empresario. Es decir, además de la explicación tradicional de que hay ciudadanos, en este caso europeos, que no quieren empresarios porque no tienen una actitud favorable hacia la actividad emprendedora se puede añadir que, incluso existiendo esa actitud favorable, también el escaso interés emprendedor puede deberse a que, en las fechas el estudio, es más atractivo ser un empleado.

El trabajo sigue la siguiente estructura: la sección 2 repasa el marco teórico de la motivación y su aplicación al fenómeno emprendedor; en la sección 3 se presenta la base de datos, las variables, la metodología y los resultados del efecto del atractivo del trabajo por cuenta ajena sobre la intención emprendedora $y$, por último, en la sección 4 se establecen las principales conclusiones y limitaciones del trabajo.

\section{MOTIVOS PARA CREAR EMPRESAS}

La motivación es una conducta dirigida y orientada hacia la consecución de metas y, por ello, se erige como una de las características fundamentales de la gente que le hace actuar de una manera concreta. Esto explica que, incluso ante situaciones idénticas, dos individuos muestren conductas muy diversas; es decir, para el caso que nos ocupa, la creación o no de una empresa. Como puntualizan Shaver y Scott (1991):

las circunstancias económicas son importantes, el marketing es importante, la financiación es importante, incluso los apoyos de agentes públicos son importantes; pero ninguno de ellos, por sí solo, crea una empresa. Para eso se necesita una persona que crea que la innovación es posible y que tenga la motivación para perseverar hasta que la tarea se ha completado. 
En la literatura reciente, los factores que se han considerado relevantes para estimular, mantener y dirigir el establecimiento de una conducta emprendedora han sido variados -Tabla 1 -.

Tabla 1: Motivadores de la actividad emprendedora

\begin{tabular}{|l|l|}
\hline Autores & Motivadores \\
\hline Veciana (1989) & $\begin{array}{l}\text { Independencia, necesidad de logro, control interno, tolerancia al } \\
\text { riesgo, insatisfacción, intuición, tolerancia de la incertidumbre, } \\
\text { capacidad de aprendizaje }\end{array}$ \\
\hline $\begin{array}{l}\text { Naffzinger, Hornsby y Kuratko } \\
\text { (1994) }\end{array}$ & $\begin{array}{l}\text { Rasgos personales, entorno personal, objetivos personales, idea de } \\
\text { negocio, entorno del negocio }\end{array}$ \\
\hline Martínez y Urbina (1998) & Dinero, afán de superación, independencia \\
\hline Rubio, Cordón y Agote (1999) & $\begin{array}{l}\text { Afán de lucro, independencia económica, independencia personal, } \\
\text { puesta en marcha de ideas propias, dirigir un grupo de personas, } \\
\text { encontrar un empleo }\end{array}$ \\
\hline $\begin{array}{l}\text { Robichaud, McGraw y Roger } \\
(2001)\end{array}$ & $\begin{array}{l}\text { Autonomía e independencia, factores intrínsecos, factores extrínsecos, } \\
\text { seguridad familiar }\end{array}$ \\
\hline Shane, Locke y Collins (2003) & $\begin{array}{l}\text { Necesidad de logro, locus de control, independencia, pasión, } \\
\text { energía, objetivos, autoeficacia }\end{array}$ \\
\hline Fernández y García (2004) & $\begin{array}{l}\text { Realización socioeconómica, espíritu inquieto, aprovechar una } \\
\text { oportunidad, ser creativo, elegir dónde y como vivir, tradición } \\
\text { familiar empresarial, desempleo, complementar baja renta }\end{array}$ \\
\hline Global Entrepreneurship Monitor & $\begin{array}{l}\text { - Oportunidad, necesidad } \\
\text { - Competencia y eficacia del emprendedor, notoriedad social, } \\
\text { estatus social, medio de hacerse rico, carrera profesional, escaso } \\
\text { miedo al fracaso }\end{array}$ \\
\hline Eurobarómetro (2004) & $\begin{array}{l}\text { Independencia y desarrollo personal, actividad interesante, } \\
\text { perspectivas de ingresos, control sobre su actividad, oportunidad } \\
\text { de negocio, evitar la incertidumbre, círculo familiar, falta de } \\
\text { empleo, coyuntura económica }\end{array}$ \\
\hline
\end{tabular}

Fuente: Elaboración propia.

A pesar de la tendencia general por la que se cree que la motivación es un rasgo personal o un conjunto de rasgos psicológicos, de manera que unas personas la tienen y otras no, este concepto es mucho más complejo y su característica distintiva es su carácter situacional; variando tanto por diferencias personales como por las diversas circunstancias en las que opera. Tal simplificación ha mostrado una cierta prevalencia en los estudios de la actividad emprendedora como conducta motivada y, consecuentemente, las teorías que consideraban que la creación de empresas depende de los rasgos personales y de la motivación del individuo emprendedor recibieron fuertes críticas por resultados empíricos contradictorios, por problemas metodológicos, por su carácter descriptivo 
y genérico y, sobre todo, por su carácter estático $0^{5}$; ya que imposibilitan la habilidad del emprendedor para aprender, desarrollar y cambiar la manera en la que gestiona sus negocios (Cope 2005). El resultado ha llevado a la tendencia opuesta: centrarse en cualquier cosa que no sea el individuo (Shaver y Scott 1991).

No obstante, este hecho es un reflejo más de la evolución general del estudio de la actividad emprendedora: la falta de consenso en una teoría general de la creación de empresas procede de la amplitud del campo de investigación, tanto por la diversidad de factores que influyen como por la existencia de distintos niveles de análisis. Su consecuencia ha sido la proliferación de estudios parciales, tanto en variables como en casos, y la creciente necesidad de buscar teorías de carácter general e integrador y estudios empíricos que aporten una visión global del proceso (Fernández y Junquera 2001). Ante tal situación, las teorías más recientes consideran que la creación de empresas es el fenómeno, a lo largo del tiempo, de la emergencia de oportunidades económicas y su explotación en nuevas empresas $\mathrm{y} / \mathrm{o}$ industrias, incorporando tanto los efectos individuales como los efectos de las oportunidades y de los entornos industrial e institucional. En consecuencia, la creación de empresas es un fenómeno multidisciplinar y complejo que no se desenvuelve únicamente en un contexto organizativo o sociológico, ni sólo consiste en una tipología de conductas, ni únicamente es un modelo de desarrollo político-económico (Shane y Venkataraman 2000; Shane 2003).

La asunción de un enfoque general multidisciplinar general y su aplicación al caso concreto de la motivación no presenta una especial complejidad conceptual; existen suficientes teorías aplicadas a la Dirección de Empresas que, adecuadamente combinadas, pueden mejorar nuestro entendimiento del fenómeno ${ }^{6}$-Figura 1 -.

5 Véase, entre otros, a Veciana (1999), Shaver (1995), Robinson et al. (1991), Stevenson y Jarrillo (1990), Gartner (1989a, 1989b, 1990), Low y McMillan (1988), Carroll y Mosakowski (1987).

Es preciso dotarse de una verdadera perspectiva psicológica en el estudio de la creación de empresas que incluya a la persona, el proceso y la elección (Shaver y Scott 1991). 
Figura 1: Principales teorías de la motivación en la Dirección de Empresas

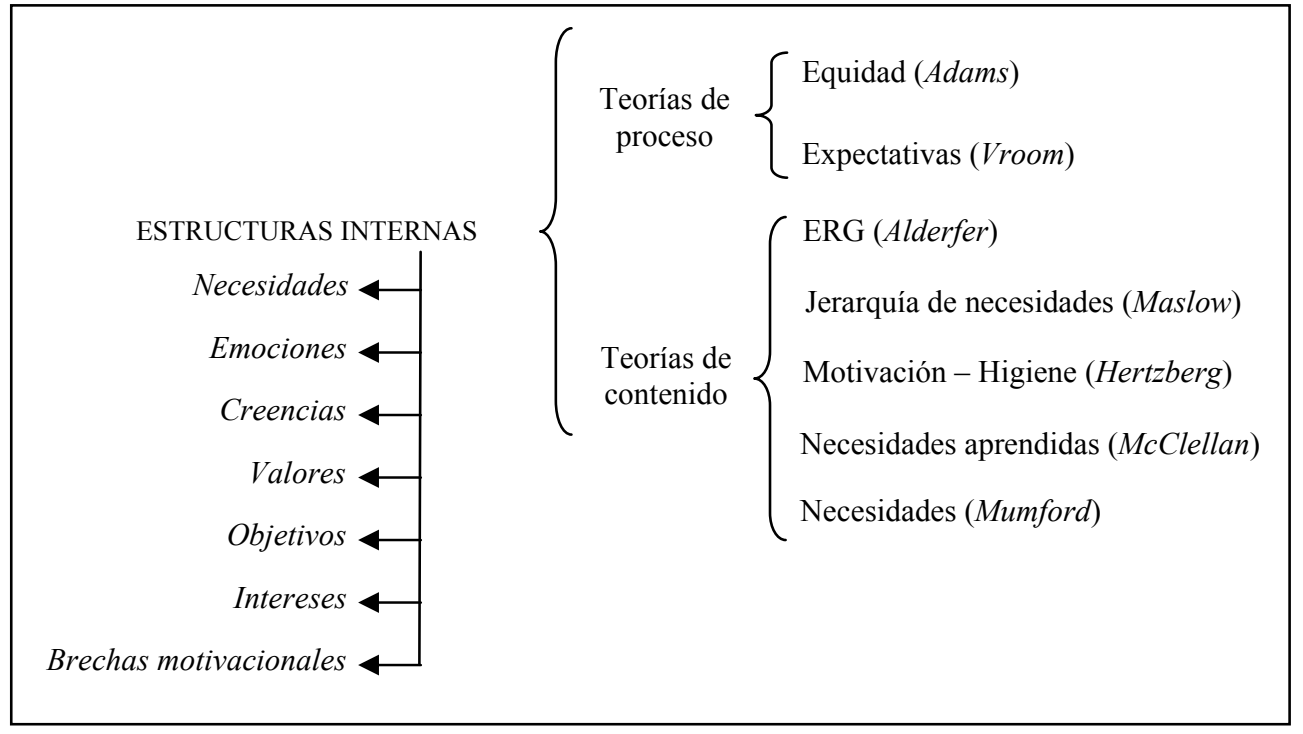

Fuente: Elaboración propia.

Tradicionalmente los estudios sobre la motivación emprendedora han utilizado como estructuras internas, fundamentalmente, rasgos de la personalidad del emprendedor y, en menor medida, características como los objetivos, expectativas y necesidades. Los trabajos más recientes, como algunos de los citados en la Figura 1, ya tratan de realizar esa integración de las estructuras internas de la motivación en teorías, bien sean de proceso bien sean de contenido. No obstante, tanto en unos como en otros, se ha relegado el estudio del efecto del "coste de oportunidad" de convertirse en empresario: el trabajo por cuenta ajena. Así, se ha considerado que hay unos motivadores, bien sean necesidades, objetivos, creencias, etcétera, exclusivamente asociados a la actitud emprendedora; de manera que la estructura interna motivacional está formada por un único factor común. Ante esta situación, el propósito de este trabajo se circunscribe en realizar una primera comparación de los motivadores del trabajo por cuenta propia y ajena como determinantes de la conducta emprendedora ${ }^{7}$; es decir, introducir el trabajo por cuenta

7 Aunque se defiende la necesidad de estudiar la actividad emprendedora y sus motivaciones desde un ámbito multidisciplinar, complejo y dinámico, en la práctica asumir o no este hecho no mediatiza el objetivo del estudio, dado su carácter exploratorio; pero, no obstante, es necesario indicarlo a efectos de matizar conclusiones y posteriores líneas de investigación. 
ajena como una estructura interna más de motivación. Consecuentemente, se espera que la motivación presente una estructura dual o bidimensional, formada dos componentes independientes: el atractivo o motivación por el autoempleo y el atractivo o motivación por el empleo por cuenta ajena. Adicionalmente, para ello, se considera una conducta proactiva bajo el esquema clásico de procesamiento cognitivo donde, ante los estímulos del entorno, el individuo construye creencias (estructuras internas de motivación), de cuya combinación se formarán, inicialmente, actitudes o valoraciones y, posteriormente, intenciones o planes de acción -Figura 2-.

Figura 2: Modelo teórico

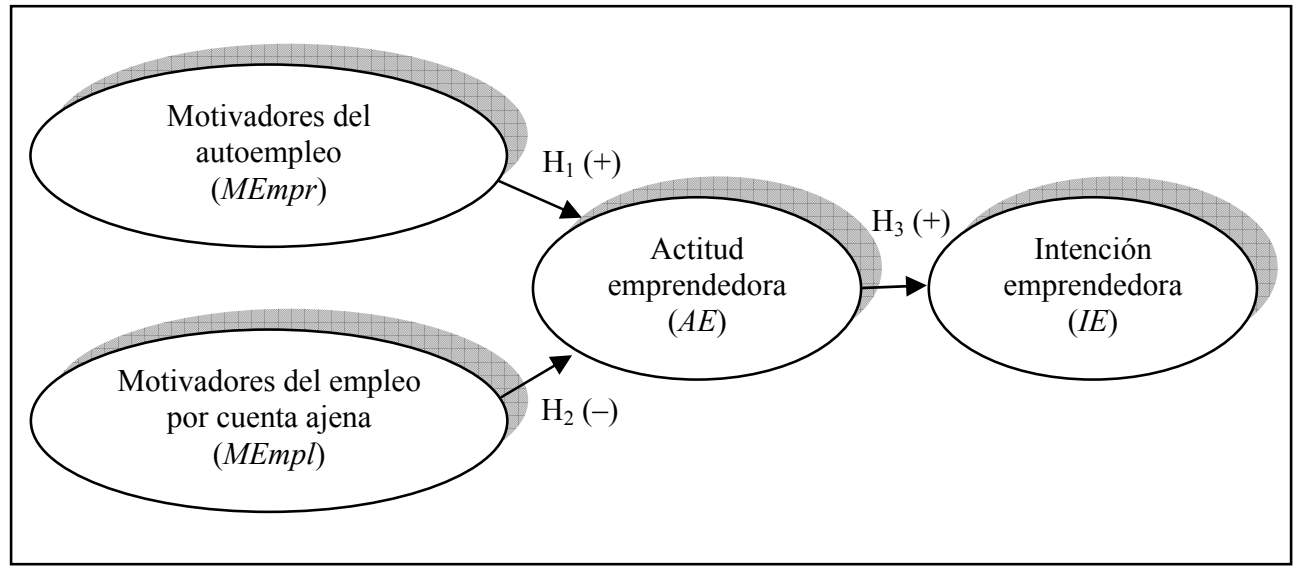

Las hipótesis que resultan de tal planteamiento son:

$\mathrm{H}_{1}$ : Existe una relación directamente proporcional entre los motivadores del autoempleo y la actitud emprendedora.

$\mathrm{H}_{2}$ : Existe una relación inversamente proporcional entre los motivadores del empleo por cuenta ajena y la actitud emprendedora.

$\mathrm{H}_{3}$ : Existe una relación directamente proporcional entre la actitud emprendedora y la intención de crear una empresa.

\section{METODOLOGÍA Y RESULTADOS}

\subsection{Datos}

Los datos empleados proceden del Eurobarómetro sobre Creación de Empresas, elaborado por la Comisión Europea, y se ha escogido 
porque recoge la información apropiada para los objetivos de este estudio: motivadores de la figura de empresario y motivadores de la figura de empleado; no obstante, la fiabilidad inicial de los datos está asegurada dado el tamaño de la muestra y los métodos de muestreo utilizados (Comisión Europea 2004). La muestra contiene los datos de un total de 18.547 ciudadanos de la Unión Europea correspondiendo a cada país un número de entrevistas ponderado proporcionalmente a la participación del mismo en la UE -Tabla 2-.

Tabla 2: Tamaño muestral

\begin{tabular}{|l|r|r|}
\hline País & Muestra & \multicolumn{1}{c|}{ \% } \\
\hline Bélgica & 419 & 2,3 \\
\hline Dinamarca & 216 & 1,2 \\
\hline Alemania & 3.428 & 18,5 \\
\hline Grecia & 443 & 2,4 \\
\hline España & 1.659 & 8,9 \\
\hline Francia & 2.390 & 12,9 \\
\hline Irlanda & 148 & 0,8 \\
\hline Italia & 2.450 & 13,2 \\
\hline Luxemburgo & 18 & 0,1 \\
\hline Países Bajos & 644 & 3,5 \\
\hline Austria & 344 & 1,8 \\
\hline Portugal & 413 & 2,2 \\
\hline Finlandia & 210 & 1,1 \\
\hline Suecia & 358 & 1,9 \\
\hline Reino Unido & 2.396 & 12,9 \\
\hline Chipre & 29 & 0,2 \\
\hline República Checa & 424 & 2,3 \\
\hline Estonia & 55 & 0,3 \\
\hline Hungría & 412 & 2,2 \\
\hline Letonia & 96 & 0,5 \\
\hline Lituania & 147 & 0,8 \\
\hline Malta & 16 & 0,1 \\
\hline Polonia & 1.545 & 8,3 \\
\hline Eslovaquia & 214 & 1,2 \\
\hline Eslovenia & 83 & 0,4 \\
\hline & 18.547 & 100 \\
\hline
\end{tabular}

\subsection{Variables}

La Tabla 3 muestra todas las variables utilizadas en el estudio y sus principales estadísticos descriptivos (media, desviación típica, valor máximo y mínimo). Dichas variables recogen creencias, objetivos y 
necesidades como motivadores de la actividad emprendedora de los ciudadanos de la Unión Europea, agrupadas porcentualmente por país.

Tabla 3: Motivadores de la actividad emprendedora

\begin{tabular}{|c|c|c|c|c|c|}
\hline \multicolumn{2}{|c|}{ Motivadores del empleo por cuenta ajena (MEmpl) } & \multirow{2}{*}{$\begin{array}{r}\text { Media } \\
33,44\end{array}$} & \multirow{2}{*}{$\frac{\text { Dt. }}{16,15}$} & \multirow{2}{*}{$\frac{\text { Max }}{67}$} & \multirow{2}{*}{$\frac{\text { Min }}{8}$} \\
\hline Q2_\$ & Regularidad ingresos & & & & \\
\hline Q2_E & Estabilidad laboral & 28,48 & 14,66 & 57 & 9 \\
\hline Q2_Ss & Seguridad social y los seguros & 12,96 & 11,49 & 53 & 1 \\
\hline Q2_NoId & Falta de idea empresarial & 5,32 & 6,01 & 28 & 0 \\
\hline Q2_NoT & Falta de tiempo & 7,16 & 5,43 & 21 & 1 \\
\hline Q2_NoI & Falta de interés & 8,96 & 8,88 & 46 & 1 \\
\hline Q2_No\$ & Falta de dinero & 8,84 & 10,49 & 43 & 1 \\
\hline Q2_NoH & Falta de habilidades & 6,12 & 4,77 & 18 & 1 \\
\hline Q2_NoC & Falta de conocimientos & 4,8 & 5,66 & 26 & 0 \\
\hline Q2_D & Dificultad de echarse atrás & 5,2 & 4,88 & 20 & 0 \\
\hline Q2_N & Nunca me lo planteé antes & 5,92 & 7,81 & 33 & 1 \\
\hline Q2_R & Aversión al riesgo y clima económico & 15,04 & 10,1 & 47 & 6 \\
\hline Q2_B & Barreras administrativas & 4,32 & 3,78 & 17 & 1 \\
\hline
\end{tabular}

\begin{tabular}{|c|c|c|c|c|c|}
\hline \multicolumn{2}{|c|}{ Motivadores del autoempleo (MEmpr) } & \multirow{2}{*}{$\begin{array}{r}\text { Media } \\
7,16\end{array}$} & \multirow{2}{*}{$\frac{\text { Dt. }}{5,43}$} & \multirow{2}{*}{$\frac{\text { Max }}{21}$} & \multirow{2}{*}{$\frac{\text { Min }}{1}$} \\
\hline Q1_In & Independencia personal & & & & \\
\hline Q1_O & Oportunidad de negocio & 8,96 & 8,88 & 46 & 1 \\
\hline Q1_E & Escoger tu entorno & 8,84 & 10,49 & 43 & 1 \\
\hline Q1_\$ & Mejores ingresos & 6,12 & 4,77 & 18 & 1 \\
\hline Q1_NoE & Falta de empleo & 4,8 & 5,66 & 26 & 0 \\
\hline Q1 NoEz & Falta de empleo en la zona & 5,2 & 4,88 & 20 & 0 \\
\hline Q1_F & Familia y/o amigos empresarios & 5,92 & 7,81 & 33 & 1 \\
\hline Q1_C & Clima económico favorable & 15,04 & 10,1 & 47 & 6 \\
\hline Q1_I & Evitar incertidumbres de ser empleado & 4,32 & 3,78 & 17 & 1 \\
\hline
\end{tabular}

\begin{tabular}{|c|c|c|c|c|c|}
\hline \multicolumn{2}{|c|}{ Actitud emprendedora $(A E)$} & \multirow{2}{*}{$\frac{\text { Media }}{42,96}$} & \multirow{2}{*}{$\frac{\text { Dt. }}{9,76}$} & \multirow{2}{*}{$\frac{\text { Max }}{62}$} & \multirow{2}{*}{$\frac{\text { Min }}{28}$} \\
\hline Q3_E & Preferencia por ser empresario & & & & \\
\hline
\end{tabular}

\begin{tabular}{|c|c|c|c|c|c|}
\hline \multicolumn{2}{|c|}{ Intención emprendedora (IE) } & \multirow{2}{*}{$\begin{array}{r}\text { Media } \\
31,72\end{array}$} & \multirow{2}{*}{$\begin{array}{l}\text { Dt. } \\
10,76 \\
\end{array}$} & \multirow{2}{*}{$\begin{array}{l}\text { Max } \\
50 \\
\end{array}$} & \multirow{2}{*}{$\begin{array}{l}\text { Min } \\
15 \\
\end{array}$} \\
\hline Q3 D & Deseabilidad de ser empresario en 5 años & & & & \\
\hline Q3_F & Factibilidad de ser en empresario en 5 años & 30,72 & 10,07 & 55 & 10 \\
\hline
\end{tabular}

\subsection{Técnica de análisis de datos y resultados}

El Análisis de Ecuaciones Estructurales (SEM) se considera como la técnica más apropiada para los objetivos del estudio puesto que estima relaciones múltiples y cruzadas y permite representar conceptos no observados o constructos (Hayr Jr. et al. 1999). Por las mismas razones, dentro de esta familia de técnicas, se ha optado por el análisis Partial 
Least Squares (PLS) ya que, además de preservar la varianza global, permite trabajar con supuestos más relajados que el análisis de estructuras de covarianzas: multicolinealidad, normalidad, tamaño muestral. En definitiva, PLS permite reflejar condiciones teóricas y empíricas de las ciencias de la conducta, donde son habituales la falta de teorías no suficientemente asentadas y la falta de información (Real et al. 2006).

Como cualquier metodología SEM, la utilización de PLS precisa de la realización de un modelo de medida y de un modelo estructural. Ambos modelos se representan en la Figura 3. El primero de ellos especifica las relaciones entre las variables observables -indicadores- con las variables latentes -conceptos teóricos o constructos-, mientras que el segundo evalúa la existencia y magnitud de las relaciones entre variables latentes (Barclay et al. 1995). Para establecer el modelo de medida se ha optado por establecer indicadores del tipo A -efecto o reflexivos- porque esas variables manifiestas u observadas se consideran causadas por los constructos latentes. La cuantía de variables latentes y manifiestas no se ha visto alterado a priori por restricciones muestrales pues el número de observaciones agrupadas (25) cumple con la heurística propuesta por Chin (1998); que considera que hay que tener una muestra 10 veces superior al número de sendas que llegan al constructo endógeno final.

Figura 3: Modelo estructural y de medida definitivo

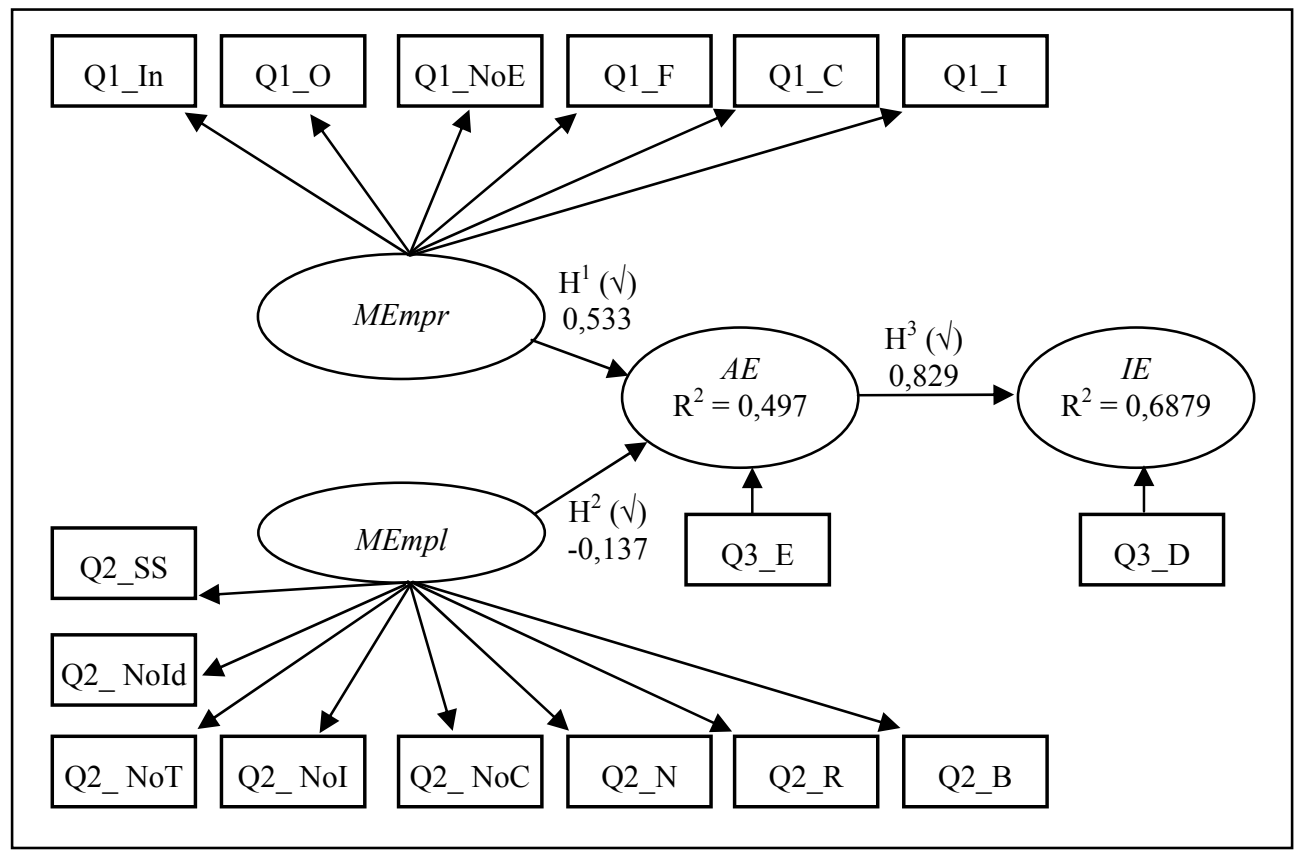


A la hora de analizar la adecuación de dicho modelo de medida es preciso comprobar: la fiabilidad individual de cada indicador $(\mathrm{FI})$, la validez convergente de los indicadores a la hora de asociarse a cada constructo (VC) y, por último, la validez discriminante (VD) (Hulland 1999) -Tablas 4 y 5-. Se consideran indicadores fiables todos aquellos superiores a 0,7 pues se justifica un $50 \%$ de la varianza común causada en cada constructo. Esto implica que hay más varianza explicada por la relación entre la variable latente y la observada que la debida al error de la varianza (Carmines y Zeller 1979). No obstante, en las etapas iniciales del desarrollo de las escalas de medida algunos autores defienden el relajo de tal norma hasta límites del 0,4 ó 0,5 (Ching 1998) pero sin olvidar que cargas demasiado bajas pueden sesgar la estimación de los parámetros (Nunnally 1978). Por todo ello, dado el objetivo del estudio, se ha tomado la decisión de eliminar todos aquellos indicadores reflectivos con cargas inferiores a 0,6. Así, para la escala de intención emprendedora se ha eliminado la variable observada Q3_F. Análogamente se han rechazado Q1_NoEz, Q1_E, Q1_\$ para la escala de actitudes hacia el autoempleo además de Q2_E, Q2_D, Q2_No\$, Q2_NoH y Q2_\$ para la escala de actitudes hacia el empleo por cuenta ajena (aunque todos los indicadores inicialmente presentan validez convergente; Alfa de Cronbach: $[\mathrm{AEmpr}]=0,8842[\mathrm{Aempl}]=0,9044[\mathrm{IE}]=0,4322)$.

Tabla 4: Fiabilidad y validez convergente del modelo de medida

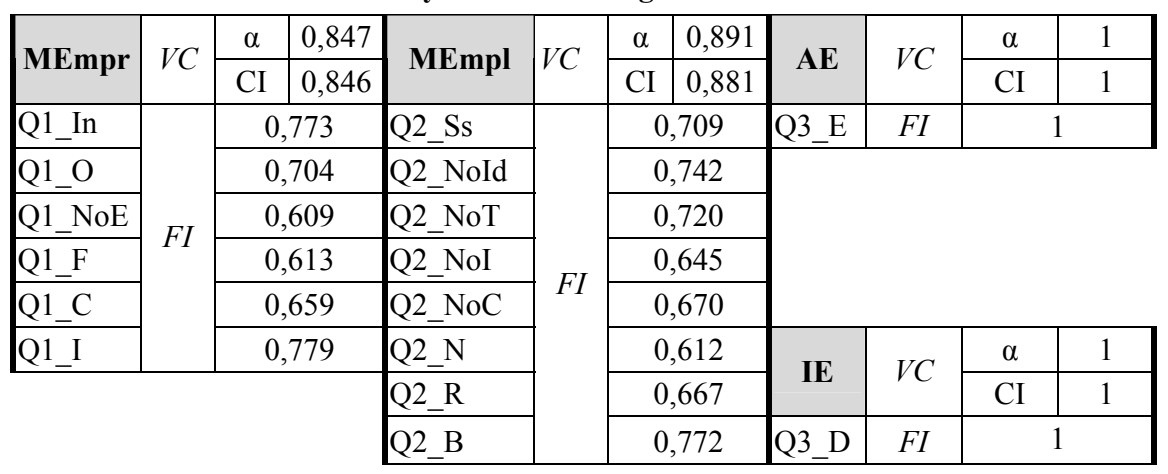

La validez convergente se puede medir tanto por medio del Alfa de Cronbach $(\alpha)$ como por la medida de consistencia interna $(\mathrm{Cl})$ de Fornell y Larcker (1981) aunque la interpretación de sus valores es similar y se considera aceptable un resultado de los test superiores a 0,7 (Nunnally 
1978). Los valores obtenidos en el estudio cumplen esta norma, luego existe validez convergente entre las variables latentes y las manifiestas.

Para terminar con la validación del modelo de medida es preciso comprobar la validez discriminante, lo que nos permite verificar que las medidas de un constructo difieren de las medidas del resto de constructos del modelo. Siguiendo a Fornell y Larcker (1981), de la comparación de la varianza promedio extraída (AVE) con las correlaciones entre las variables latentes se puede confirmar la validez discriminante de los constructos propuestos (aunque es preciso destacar que la alta correlación observada entre los indicadores motivadores ha permitido validar el resultado sólo por un escaso margen).

Tabla 5: Validez discriminante del modelo de medida

\begin{tabular}{|l|c|c|c|c|}
\hline Constructos & MEmpr & Mempl & AE & IE \\
\hline MEmpr & $1(A V E=0,480344)$ & & & \\
\hline Mempl & 0,683375 & $1(A V E=0,481172)$ & & \\
\hline AE & 0,398795 & $-0,387652$ & $1(A V E=1)$ & \\
\hline IE & 0,461974 & $-0,454667$ & 0,829424 & $1(A V E=1)$ \\
\hline
\end{tabular}

Puesto que PLS trata de minimizar el error, maximizar la varianza explicada, no existe una medida de la bondad del ajuste global de las relaciones entre las variables latentes y la validez del modelo estructural ha de asegurarse mediante el examen de los valores R2 de cada uno de los constructos endógenos del modelo, siendo un valor igual o superior a 0,5 considerado como aceptable. En la Figura 3, se puede apreciar que ambas variables latentes endógenas del modelo pueden considerarse que cumplen este criterio $^{8}$ pero, para el caso de la Actitud Emprendedora, este ajuste está en los límites tolerables.

Una vez que se ha verificado la validez del modelo de medida y del modelo estructural se puede afirmar que las hipótesis planteadas se han contrastado; en la Figura 3 se indican los coeficientes path estandarizados $[\mu=0 ; \sigma=1]$ que recogen el efecto de cada contraste 9 . Estos resultados son consecuentes con las teorías cognitivas del

\footnotetext{
8 Adicionalmente todos las sendas (paths) poseen valores de la $\mathrm{T}$ de Student superiores a 1,96 .

Aún así es preciso tener en cuanta que la aproximación PLS sustituye la noción de causalidad por la de predictibilidad (Falk y Miller 1992).
} 
comportamiento. Así, la tercera hipótesis ratifica una vez más que la conducta (creación de una empresa) precisa un primer impulso inicial (intención emprendedora) formado por las valoraciones que el individuo realiza sobre la idoneidad de tal conducta (actitud emprendedora) $\left[\mathrm{H}_{3}\right.$ : $B=0,829 ; p<0,001]$. Esas valoraciones son fruto de un conjunto de creencias diversas, que actúan como motivadores iniciales de la conducta, y que los resultados permiten concluir que se pueden agrupar en dos categorías: motivadores iniciales $\mathrm{o}$ atractivo del autoempleo $\left[\mathrm{H}_{2}\right.$ : $B=0,533 ; p<0,001]$ y motivadores iniciales $o$ atractivo del empleo por cuenta ajena $\left[H_{1}: B=-0,137 ; p<0,001\right]$.

\section{CONCLUSIONES Y LIMITACIONES}

\subsection{Conclusiones}

En la literatura sobre motivación emprendedora tradicionalmente se han considerado diversos elementos estructurales internos, predominantemente creencias, objetivos e intereses. Pero, independientemente del tipo concreto estructural sobre el que se ha medido el fenómeno, también se aprecia que todos ellos han sido seleccionados considerando que forman parte de una única dimensión intrínseca al hecho de convertirse en empresario. De esta forma la motivación emprendedora estaría formada por todos aquellos rasgos estructurales internos asociados a ser empresario y de su mayor o menor valoración por el individuo surge una mayor o menor motivación hacia convertirse efectivamente en empresario (emprendedor será entonces quien tenga valoraciones positivas de las consecuencias de crear una empresa y viceversa).

La evidencia empírica muestra un relativo escaso interés emprendedor de los individuos, ¿significa esto, según lo recién citado, que la figura del emprendedor es abrumadoramente denostada?. Tal evidencia empírica, por ejemplo de paneles como los del GEM o el Eurobarómetro, sí muestra el escaso interés e incluso una relativa baja valoración de la figura del emprendedor pero no tan acusada como para justificar tasas finales de motivación tan bajas. Es por ello que el estudio ha tratado de responder a preguntas que intuitivamente aparecen: ¿hay casos en los que alguien con valoraciones iniciales positivas hacia la actividad emprendedora puede decidir no convertirse en empresario?, 
¿existen factores ajenos al hecho de convertirse en emprendedor que afecten a su atractivo motivador?.

Los resultados muestran que sí existen factores ajenos y que se relacionan con el coste de oportunidad de desarrollar una carrera profesional como empresario, con la opción alternativa de ser un empleado por cuenta ajena. Los elementos estructurales internos intrínsecamente asociados con el hecho de ser un empleado también influyen, en este caso negativamente, en la valoración del atractivo de convertirse en empresario. La no existencia de espíritu emprendedor no procede únicamente de valoraciones negativas sobre la actividad emprendedora sino que aún en el caso de la existencia de valoraciones positivas esos valores se ven minorados por la valoración del atractivo de ser empleado, de manera que la opción final preferida no sea el autoempleo.

Desde un punto de vista práctico, se puede plantear que las políticas de estímulo de la actividad emprendedora deberían tener en cuenta que no exclusivamente los ciudadanos con actitudes negativas hacia el autoempleo rechazan crear empresas. Dado que el atractivo del empleo por cuenta ajena es otro determinante adicional significativo, los programas de educación para el fomento del espíritu emprendedor podrían ser más efectivos si la ponderación de las bondades de la actividad emprendedora se hiciese también en relación con los aspectos ajenos a la misma pero que fomentan el empleo por cuenta ajena (dado que no todos los motivadores funcionan de manera opuesta para ambos tipos de empleo).

\subsection{Limitaciones}

Debido a razones operativas de acceso a la información, este estudio comparte una limitación habitual en los estudios de motivación emprendedora: la mezcla de indicadores relacionados con distintas estructuras internas. Aunque para los objetivos del estudio, verificar la existencia de una estructura dual, es probable que esto no afecte a los resultados obtenidos (el modelo planteado explica un $68 \%$ de la varianza de la intención emprendedora) una primera manera de mejorar el conocimiento del fenómeno sería el tratamiento de los motivadores bajo una adecuada taxonomía de elementos estructurales (necesidades, emociones, creencias, valores, objetivos, intereses...). Así, quizá también se pueda mitigar el principal problema hallado en el modelo propuesto, la validez discriminante de los constructos motivacionales y su ajuste estructural aceptados por un escaso margen (a pesar de que la validez 
convergente de cada escala, tanto inicialmente como tras estimar el modelo, era alta).

Otra explicación adicional de tal problema puede ser que, además de la clasificación de motivadores, haya variables observadas que reflejan extremos opuestos de un mismo estímulo o que los estímulos no tengan la misma importancia o se presenten en el mismo orden. Esto podría analizarse mediante la realización de estudios basándose en teorías concretas de motivación: bien sean de proceso, de contenido o una adecuada combinación de ambas (preferentemente con un enfoque multidisciplinar).

La muestra cumplía los requisitos de la aproximación utilizada $y$, aunque se podría haber llevado a cabo un remuestreo (por ejemplo, jackknife o, mejor aún, boostrap), para los objetivos del estudio no era preciso una reespecificación muestral. No obstante, también quizá se pueda mejorar la validez predictiva del modelo mediante la réplica del estudio en muestras mayores.

La motivación es una conducta orientada a una meta, es un concepto esencialmente dinámico que supone la existencia de un impulso inicial a actuar y de unos impulsos posteriores que perseveren en la continuidad de la tarea hasta lograr el objetivo propuesto. Este estudio es estático, se limita a establecer la existencia de una estructura dual pero sólo en el momento inicial. Indudablemente es preciso realizar estudios longitudinales que analicen el potencial mantenimiento de esta estructura a lo largo de todo el proceso y la posible identificación de etapas o de variaciones en efectos motivadores.

\section{BIBLIOGRAFÍA}

BAUMOL, W.J. (1993) "Formal Entrepreneurship Theory in Economics: Existence and Bounds", Journal of Business Venturing, vol. 8, $\mathrm{n}^{\circ} 2$, pp. 197-210.

- (2004) "Entrepreneurial Cultures and Countercultures", Academy of Management Learning and Education, vol. 3, $\mathrm{n}^{\circ} 3$, pp. 316-326.

BARCLAY, D.; C. HIGGINS and R. ThOMSON (1995) "The Partial Least Squares (PLS) Approach to Causal Modelling: Personal Computer Adoption and Use as Illustration", Technological Studies, vol. 2, n² 2, pp. 285-309. 
BRoWN, T.E. \& J.M. ULJIN (2004) Innovation, Entrepreneurship and Culture: the Integration Between Technology, Progress and Economic Growth. Cheltenham (Reino Unido): Edward Elgar Publishers.

CARMINES, E.G. \& R.A. ZeLler (1979) "Reliability and Validity Assessments", Sage University Paper Series on Quantitative Applications in the Social Sciences, vol. 7, $\mathrm{n}^{\circ}$ 07, Sage, Beverly Hills (EE. UU.).

CARROLL, G. \& E. MOSAKOWSI (1987) "The Career Dynamics of SelfEmployment", Administrative Science Quarterly, vol. 32, $\mathrm{n}^{\circ} 4$, pp. 570589.

CHIN, W.W. (1998) "The Partial Least Squares Approach to Structural Equation Modeling". G.A. MARCOULIDES (ed.) Modern Methods for Business Research. Mahwah: Lawrence Erlbaum Associates, pp. 295-336.

COMISIÓN EUROPEA (2004) Flash Eurobarometer (160): Entrepreneurhip. Bruselas: Comisión Europea.

COPE, J. (2005) "Toward a Dynamic Learning Perspective of Entrepreneurship", Entrepreneurship: Theory \& Practice, vol. 29, $\mathrm{n}^{\circ} 4$, pp. 373-374.

CUERVO, A. (2003) "La creación empresarial: de empresarios y directivos". Enric GeNesCá, David URBANo, Joan Lluís CAPELleRAS, Carlos GuallaRTE y Joaquín VERGÉs (2003) Creación de Empresas: homenaje al profesor Josá María Veciana Vergés. Barcelona: UAB, pp. 49-74.

ECKHARDT, J. \& S. SHANE (2003) "The Importance of Opportunities to Entrepreneurship", Journal of Management, vol. 29, n 3, pp. 333-349.

FALK, R.F. \& N.B. MILleR (1992) A Primer for Soft Modelling. Akron (EE. UU.): University of Akron.

FERNÁNDEZ, E. y B. JUNQUERA (2001) "Factores determinantes en la creación de pequeñas empresas: una revisión de la literatura", Papeles de Economía Española, n $89 / 90$, pp. 322-342.

FERNÁNDEZ, W. y A. GARCíA (2004) "Motivaciones y obstáculos en función de la experiencia empresarial del emprendedor", Actas del XIV Congreso de la Asociación Científica de Economía y Dirección de Empresa (ACEDE), 19-21 septiembre, Murcia.

FORNELL, C. y D.F. LARCKER (1981) "Evaluating Structural Equation Models with Unobservable Variables and Measurement Error", Journal of Marketing Research, $\mathrm{n}^{\circ} 18$, pp. 39-50.

GARTNER, W.B. (1989a) "«Who is an entrepreneur?» is the wrong question", American Journal of Small Business, vol. 13, $\mathrm{n}^{\circ} 4$, pp. 43-69. 
- (1989b) "Some Suggestions for Research on Entrepreneurial Traits and Characteristics", Entrepreneurship: Theory \& Practice, vol. 14, $\mathrm{n}^{\circ} 1$, pp. 27-37.

- (1990) "What Are We Talking About When We Talk About Entrepreneurship?", Journal of Business Venturing, vol. 5, $n^{\circ} 1$, pp. 1528.

Genescá, E.; D. Urbano, J.L. Capelleras, C. Guallarte y J. Vergés (2003) Creación de Empresas: homenaje al profesor Josá María Veciana Vergés. Barcelona: UAB.

HAIR JR, J.F.; R.E. ANDERSON, R.L. TATHAM y W.C. BlaCK (1999) Análisis multivariante ( $5^{\mathrm{a}}$ edición). Madrid: Prentice Hall.

HoRnADAY, R.W. (1992) "Thinking About Entrepreneurship: A Fuzzy Set Approach", Journal of Small Business Management, vol. 30, $\mathrm{n}^{\circ} 3$, pp. 12-23.

HULLAND, J. (1999) "Use of Partial Least Squares (PLS) in Strategic Management Research. A Review of Four Recent Studies", Strategic Management Journal, $\mathrm{n}^{\circ}$ 20, pp. 195-204.

INSTITUTO DE EMPRESA (2004) Global Entrepreneurship Monitor. Informe Ejecutivo (2004) - España. Madrid.

LOW, M.B. \& I.C. MCMILLAN (1988) "Entrepreneurship: Past Research and Future Challenges", Journal of Management, vol. 14, $n^{\circ}$ 1, pp. 110-131.

MCGRATH, R.G. (1999) "Falling Forward: Real Options Reasoning and Entrepreneurial Failure", Academy of Management Review, vol. 24, $\mathrm{n}^{\circ}$ 1, pp. 13-30.

MARTÍNEZ, A. y O. URBINA (1998) "Emprendedores y empresas de alta tecnología: el caso de Aragón", Revista Europea de Dirección y Economía de la Empresa, vol. 7, n 3, pp. 135-146.

NAFFZIGER, D.W.; J.S. HORNSBY \& D.F. KURATKO (1994) "A Proposed Research Model of Entrepreneurial Motivation", Entrepreneurship: Theory and Practice, vol. 18, $\mathrm{n}^{\circ} 3$, pp. 29-42.

Nunnally, J.C. (1978) Psychometric Theory (2nd ed.). New York (EE.UU.): McGraw Hill.

ReAL, J.C.; A. LEAL y J.L. RoLdAN (2006) "Aprendizaje organizativo y tecnologías de la información: influencia en el desarrollo de competencias distintivas tecnológicas y resultados empresariales", Revista Europea de Dirección y Economía de la Empresa, vol. 15, n 4, pp. 9-32.

Reinolds, P.; N. Bosma, E. Autio, S. Hunt, N. de Bono, I. Servais, P. LópezGARCÍA \& N. CHIN (2005) "Global Entrepreneurship Monitor: Data 
Collection, Design and Implementation", Small Business Economy, vol. 24, n² 2, pp. 205-231.

Robichaud, Y.; E. MCGRaW \& A. Roger (2001) "Toward the Development of a Measuring Instrument for Entrepreneurial Motivation", Journal of Developmental Entrepreneurship, vol. Aug (6), n² 2, pp. 189-201.

RoBinson, P.; D. StIMPSON, J. HUEFNER \& H. HUNT (1991) "An Attitude Approach to the Prediction of Entrepreneurship", Entrepreneurship: Theory \& Practice, vol. 15, $\mathrm{n}^{\circ}$ 4, pp. 13-31.

RUBIO, E.A.; E. CORDón y A.L. AGOTE (1999) "Actitudes hacia la creación de empresas: un modelo explicativo", Revista Europea de Dirección y Economía de la Empresa, vol. 8, n 3, pp. 37-51.

Shane, S. \& S. Venkataraman (2000) "The Promise of Entrepreneurship as a Field of Research", Academy of Management Review, vol. 25, $\mathrm{n}^{\circ}$ 1, pp. 217-226.

- (2003) A General Theory of Entrepreneurship: The Individual-Opportunity Nexus. Cheltenham (Reino Unido): Edward Elgar Publishers.

-; E.A. LOCKE \& C.J. ColLINS (2003) "Entrepreneurial Motivation", Human Resource Management Review, vol. 13, $\mathrm{n}^{\circ} 2$ (summer), pp. 257-279.

SHAVER, K. \& L.R. SCOTT (1991) "Person, Process, Choice: The Psychology of New Venture Creation", Entrepreneurship: Theory \& Practice, vol. 16, $\mathrm{n}^{\circ} 2$, pp. 23-45.

- (1995) "The Entrepreneurial Personality Myth", Business and Economic Review, vol. 41, $\mathrm{n}^{\circ} 2$, pp. 20-23.

STEVENSON, H.H. \& J.C. JARILLO (1990) "A Paradigm of Entrepreneurship: Entrepreneurial Management", Strategic Management Journal, vol. 1, $\mathrm{n}^{\circ} 1$, pp. 17-27.

VeCiAnA, J.M. (1989) "Características del empresario en España", Papeles de Economía Española, $\mathrm{n}^{\circ}$ 39, pp. 19-36.

- (1999) "Creación de empresas como programa de investigación científica", Revista de Europea de Dirección y Economía de la Empresa, vol. 8, nº 3, pp. 11-33. 